\title{
Expresión de alfa sinucleína en sangre y su relación con el estreñimiento crónico en población residente en Bogotá, D.C., con problemas de consumo de alcohol
}

\author{
Tania Yadira Martínez-Rodríguez¹, Mauricio Rey-Buitrago² \\ ${ }^{1}$ Maestría en Fisiología, Departamento de Ciencias Fisiológicas, Facultad de Medicina, \\ Universidad Nacional de Colombia, Bogotá, D.C., Colombia \\ ${ }^{2}$ Maestría en Genética Humana, Departamento de Morfología, Facultad de Medicina, \\ Universidad Nacional de Colombia, Bogotá, D.C., Colombia
}

Introducción. El consumo excesivo de alcohol resulta en neuroadaptación, neurodegeneración y expresión diferencial de numerosos genes.

Objetivo. Determinar la relación entre la expresión del gen de la alfa sinucleína ( $S N C A)$ en sangre, las variantes de nucleótido único (Single Nucleotide Variant, SNV) en su región promotora y el estreñimiento crónico en personas con problemas de consumo de alcohol. Materiales y métodos. La muestra estuvo conformada por 35 controles y 27 casos, seleccionados según el puntaje obtenido con la herramienta AUDIT. En el diagnóstico del estreñimiento se aplicaron los criterios de Roma IV. La extracción de ácidos nucleicos se hizo a partir de sangre periférica y se evaluó la expresión del gen mediante qPCR, la cuantificación proteica por ELISA y la presencia de SNV en la región promotora del gen por la secuenciación de Sanger.

Resultados. Se observó sobreexpresión génica relativa de ARNm del gen SNCA en el grupo de casos sin relación con el estreñimiento crónico. Se evidenció un riesgo 4,8 veces mayor de presentar estreñimiento en el grupo de casos. Se encontraron nueve variantes de nucleótido simple en un segmento de la región promotora del gen rica en secuencias reguladoras $\mathrm{CpG}$, con frecuencia similar entre los grupos, y se detectó una variante en la posición -2171 que no se encuentra reportada en GenBank para variantes clínicas y cuyo genotipo A/T se relacionó con el incremento de la expresión del ARNm del SNCA. Conclusión. En personas con problemas de consumo de alcohol se evidenció la

Recibido: $15 / 11 / 2018$

Aceptado: $12 / 09 / 2019$

Publicado: 13/09/2019

\section{Citación:}

Martínez-Rodríguez TY, Rey-Buitrago M. Expresión de alfa sinucleína en sangre y su relación con el estreñimiento crónico en población residente en Bogotá, D.C. con problemas de consumo de alcohol. Biomédica. 2020;40:309-21.

https://doi.org/10.7705/biomedica.4771

\section{Correspondencia:}

Mauricio Rey-Buitrago, Instituto de Genética, Universidad Nacional de Colombia, Carrera 45 No 26-85, edificio 426, Bogotá, D.C., Colombia Teléfono: (571) 316 5000, extensión 11610 mrey@unal.edu.co

Contribución de los autores:

Ambos autores participaron en todas las etapas de la investigación.

\section{Financiación:}

Esta publicación es producto de los compromisos adquiridos en la convocatoria interna de proyectos para el fortalecimiento de la investigación, creación e innovación de la Universidad Nacional de Colombia, 2016-2018, con el proyecto titulado: "Estudio de genes de neuroinflamación, su expresión y estado de metilación en sujetos alcohol-dependientes colombianos", código Hermes 34776.

\section{Conflicto de intereses:}

Los autores declaramos no tener conflictos de intereses. sobreexpresión del ARNm de alfa sinucleína, lo cual no se relacionó con el diagnóstico de estreñimiento crónico.

Palabras clave: alcoholismo; estreñimiento; alfa-sinucleína; expresión génica; polimorfismo genético; inflamación.

Alpha sinuclein expression in blood and its relationship with chronic constipation in a population from Bogotá, D.C., with problems of alcohol consumption

Introduction: Excessive alcohol consumption results in neuroadaptation, neurodegeneration, and differential expression of numerous genes.

Objective: To determine the relationship between the expression of the alpha synuclein gene (SNCA) in blood, single nucleotide variant (SNV) in its promoter region, and chronic constipation in people with problems of alcohol consumption.

Materials and methods: The sample consisted of 35 controls and 27 cases selected according to the score obtained with the AUDIT tool. For the diagnosis of constipation, the Rome IV criteria were applied. Nucleic acid extraction was performed from peripheral blood and the expression of the gene was evaluated by qPCR, protein quantification by ELISA, and the presence of SNV in the promoter region of the gene by Sanger sequencing. Results: We observed a relative gene overexpression of SNCA mRNA in the case group, which was not related to the diagnosis of chronic constipation. There was 4.8 times greater risk of presenting constipation in the group of cases. Besides, nine single nucleotide variants were found in a segment of the promoter region of the gene rich in CpG regulatory sequences with similar frequency between the groups while a variant was identified in position -2171, which is not reported in GenBank for variants and whose genotype A/T was associated with increased expression of SNCA mRNA.

Conclusion: We evidenced an overexpression of alpha synuclein mRNA in people with problems of alcohol consumption that was not related to the diagnosis of chronic constipation.

Keywords: Alcoholism; constipation; alpha synuclein; gene expression; genetic polymorphism; inflammation. 
A nivel mundial se estima que anualmente el abuso del alcohol causa más de dos millones de muertes y diferentes enfermedades agudas y crónicas, incrementando los costos de la atención en salud y comprometiendo el desarrollo del individuo, la familia y la comunidad (1). En Colombia cerca de dos millones y medio de personas consumen alcohol en niveles perjudiciales, lo que representa el $31 \%$ de los consumidores de alcohol y el $11 \%$ de la población entre los 12 y los 65 años (2).

El alto consumo de alcohol causa neurotoxicidad y neuroadaptación (37). Se ha postulado que la proteína alfa sinucleína (SNCA) está implicada en la sinapsis, la plasticidad neuronal y diversas funciones en las neuronas dopaminérgicas, lo cual la vincula con el sistema de recompensa cerebral que se ve alterado en las adicciones, especialmente el alcoholismo (3). Además, se ha demostrado que el flujo de SNCA adopta la dirección cerebro-sangre como mecanismo regulador (8), lo que permite su detección en los fluidos humanos (9-16). En este sentido, los estudios en este campo reportan la expresión diferencial del gen que codifica para dicha proteína asociada con la búsqueda y el deseo compulsivo de alcohol (craving) (17); asimismo, en los estudios en ratas y primates no humanos se ha evidenciado que la sobreexpresión de alfa sinucleína en sangre resulta del consumo prolongado de alcohol $(18,19)$, lo que ha permitido concluir que la elevación de los niveles de ARNm en sangre es común en humanos, roedores y primates y que la alfa sinucleína podría ser útil como biomarcador periférico de alcoholismo crónico (17-19).

Por otro lado, el gen SNCA es muy polimorfo, incluida la región promotora participante en el proceso de inicio y regulación de la transcripción; algunas de estas variantes se han relacionado con el alto consumo de alcohol. El polimorfismo Rep1 en la SNCA se asocia con fenotipos de uso, abuso, dependencia y búsqueda de alcohol (20); también se han identificado cinco SNV en esta región promotora del gen: rs7678651(C>A), rs7687945 (C>A,T), rs2736995 (A>C), rs2619364 (A>C,G), y rs2301134 $(A>G)$, asociados con el fenotipo del deseo compulsivo (craving) de alcohol (21).

Considerando la influencia de la SNCA en la actividad de la dopamina y las funciones de este neurotransmisor en la motilidad, se han establecido hipótesis sobre una posible relación entre el intestino y el cerebro que postulan que la acumulación de alfa sinucleína se inicia en el intestino y se propaga a través del sistema nervioso entérico hasta el sistema nervioso central $(22,23)$, observándose la disminución de la motilidad intestinal y la aparición de estreñimiento en los modelos animales (24-30).

En este sentido, asumiendo una respuesta similar en humanos y dado el común denominador de la SNCA con el alcoholismo y el estreñimiento, el objetivo del estudio fue determinar la relación entre la expresión de la alfa sinucleína en sangre, las variantes de nucleótido único (SNV) en la región promotora del gen y el estreñimiento crónico en una muestra de población residente en Bogotá con problemas de consumo de alcohol.

\section{Materiales y métodos}

\section{Muestra de estudio}

Se llevó a cabo un estudio exploratorio observacional del tipo de casos y controles con una muestra seleccionada a conveniencia. Participaron inicialmente 306 sujetos residentes en Bogotá convocados por las redes sociales de la Universidad Nacional de Colombia, que respondieron la 
encuesta inicial para la aplicación de los criterios de inclusión y exclusión, así como el cuestionario Alcohol Use Disorders Identification Test (AUDIT) después de firmar el debido consentimiento informado.

Según el puntaje del AUDIT, 115 sujetos cumplían con el correspondiente al grupo de control ( $\leq 2$ puntos) o al grupo de casos ( $\geq 16$ puntos), es decir, quienes consumían alcohol en niveles perjudiciales o de dependencia. Estos fueron citados para la segunda fase de toma de las muestras de sangre, a la que asistieron solamente 62 individuos ( 35 sujetos en el grupo control y 27 en el grupo de casos), que conformaron la muestra final. Se excluyeron aquellos sujetos que consumían fármacos asociados con el estreñimiento, tenían antecedentes de enfermedad hepática o un esquema de tratamiento del alcoholismo o enfermedades psiquiátricas.

\section{Diagnóstico de estreñimiento crónico}

Se aplicaron los criterios de Roma IV para el diagnóstico del estreñimiento (31); con el fin de descartar el síndrome de intestino irritable se aplicaron también los criterios de Roma IV para esta enfermedad, naturalmente teniendo en cuenta la información de la historia clínica de los participantes.

\section{Toma de muestras}

Los participantes se abstuvieron de consumir alcohol durante 48 horas como mínimo antes de la extracción de sangre periférica por venopunción. La sangre se recolectó en tubos de tapa lila con EDTA y de los $15 \mathrm{ml}$ obtenidos, seis se destinaron para el aislamiento de células mononucleares y nueve para la extracción del ADN. El plasma obtenido mediante el gradiente de densidad se conservó a $-80^{\circ} \mathrm{C}$ para su empleo en la prueba de ELISA para proteínas.

\section{Análisis de la expresión génica de ARNm}

Para analizar la expresión de ARNm se aislaron las células mononucleares mediante el gradiente de densidad con el reactivo Histopaque-1077 $7^{\mathrm{TM}}$ (Sigma-Aldrich), en tanto que la extracción de ARN total se hizo con el estuche de extracción PureLink RNA mini kit ${ }^{T M}$ (Thermo Fischer Scientific) o con el método trizol-cloroformo usando el reactivo RiboZol ${ }^{\mathrm{TM}}$. Para la reacción de transcripción inversa se utilizó el estuche High Capacity cDNA Reverse Transcription Kit ${ }^{\mathrm{TM}}$ (Thermo Fischer Scientific) con las siguientes condiciones: 1) a $25^{\circ} \mathrm{C}$ durante 10 minutos; 2) a $37^{\circ} \mathrm{C}$ durante 120 minutos; 3) a $85^{\circ} \mathrm{C}$ durante 5 minutos, y 4) a $4{ }^{\circ} \mathrm{C} \infty$.

Por último, la expresión de los genes se evaluó mediante qPCR utilizando una concentración de $1 \mathrm{ng} / \mu \mathrm{l}$ para todas las muestras de ADNc dentro del rango dinámico estandarizado, con una eficiencia de amplificación del SNCA de 2,18, y los iniciadores de diseño propio F: GCCAAGGAGGGAGTTGTGGCTGC y R: TGTTGCCACACCATGCACCACTCC, en una concentración de $0,125 \mu \mathrm{M}$, mediante la herramienta primer-blast disponible en http://www.ncbi.nlm.nih.gov/tools/primer-blast.

Para el caso del gen normalizador gliceraldehído-3-fosfato deshidrogenasa (GADPH), se obtuvo una eficiencia de 1,98 y se utilizaron los iniciadores estándar F: CACCAGGGCTGCTTTTAACTCTGGTA y R: CCTTGACGGTGCCATGGAATTTGC en una concentración de 0,25 $\mu \mathrm{M}$. Además, se utilizó una muestra calibradora interplaca obtenida de una mezcla de iguales proporciones de ADNc del grupo de casos y del de controles. Para la reacción se usó el estuche Kit Luna Universal qPCR Master Mix ${ }^{\mathrm{TM}}$; cada 
muestra se procesó por triplicado (técnico) bajo las siguientes condiciones: 1) un ciclo de desnaturalización inicial a $95^{\circ} \mathrm{C}$ durante 300 segundos; 2) 45 ciclos de desnaturalización a $95^{\circ} \mathrm{C}$ durante 15 segundos y una amplificación a 65 ${ }^{\circ} \mathrm{C}$ durante 30 segundos; 3 ) un ciclo de fusión a $95^{\circ} \mathrm{C}$ durante 10 segundos, a $65^{\circ} \mathrm{C}$ durante 60 segundos y a $97^{\circ} \mathrm{C}$ durante 1 segundo; 4) un ciclo de enfriamiento a $37^{\circ} \mathrm{C}$. Por último, con los niveles de eficiencia de cada gen y los valores de $\mathrm{Cq}$ obtenidos se hizo la conversión a la tasa de expresión.

\section{Ensayo de inmunoabsorción ligado a enzimas (ELISA)}

Para la cuantificación de proteínas se utilizó el estuche Alpha-synuclein (SNCA) (Human) ELISA ${ }^{\mathrm{TM}}$ de BioVision Incorporated, con una sensibilidad de $<9,375 \mathrm{pg} / \mathrm{ml}$ y un rango de detección entre 15,6-1.000 pg/ml. La lectura se hizo a $450 \mathrm{~nm}$ con una ventana de tiempo de 20 minutos en un equipo Multiskan $\mathrm{FC}^{\mathrm{TM}}$ de Thermo Scientific. Cada muestra se procesó por triplicado y la curva estándar se preparó con una serie de 8 diluciones de $300 \mu \mathrm{l}$ y concentraciones de $1.000,500,250,125,62,5,32,1$ y $15,6 \mathrm{pg} / \mathrm{ml}$.

\section{Evaluación de variantes de nucleótido único}

La extracción de ADN se hizo mediante la técnica de precipitación salina (salting out). Para la identificación de las SNV de la región promotora del SNCA se empleó PCR convencional con los iniciadores de diseño propio empleando la herramienta primer-blast disponible en http://www.ncbi.nlm.nih. gov/tools/primer-blast. La secuencia de los iniciadores fue la siguiente:

F: CCGCTTGTTTTAGACGGCTG y R: GTCACGAGCACTCTTGTGGA, para un segmento de $561 \mathrm{pb}$ comprendido entre los nucleótidos -1738 y -2299 de la región promotora del gen.

Después de la PCR, se purificaron los productos con acetato de sodio 3M y etanol absoluto para luego hacer la secuenciación con el método de Sanger empleando el iniciador directo, por lo que se obtuvo la secuencia de la hebra no codificante. Los electroferogramas se visualizaron con el programa BioEdit Sequence Alignment Editor disponible en http://www.mbio.ncsu.edu/BioEdit/ bioedit.html, y el alineamiento de las secuencias obtenidas con la secuencia de referencia se hizo con la herramienta BLAST2 disponible en https://blast. ncbi.nlm.nih.gov/Blast.cgi.

\section{Análisis de datos}

El análisis estadístico se hizo con los programas SPSS ${ }^{\mathrm{TM}}$ (SPSS Inc., Released 2009, PASW Statistics for Windows, version 18.0; Chicago: SPSS Inc.) y GraphPad Prism ${ }^{\mathrm{TM}}$ (GraphPad Software, Inc). Para determinar la distribución de datos se utilizaron las pruebas de Shapiro Wilk y de Kolmogorov-Smirnov. El nivel de significación se estableció en $\alpha=0,05$ y todas las pruebas se realizaron a dos colas. Se emplearon, asimismo, las pruebas de Mann-Whitney, ANOVA de dos factores con test post hoc de Bonferroni, de Friedman y el test exacto de Fisher y el de ji al cuadrado.

\section{Consideraciones éticas}

El presente estudio fue aprobado por el Comité de Ética de la Facultad de Medicina de la Universidad Nacional de Colombia mediante acta de evaluación 014-224B-17 del 28 de septiembre de 2017. Todos los sujetos aceptaron participar voluntariamente en el estudio y firmaron un consentimiento informado. 


\section{Resultados}

\section{Descripción de los sujetos de estudio}

La media de la edad de los participantes fue de $24 \pm 4$ años para el grupo de control y de $23 \pm 4$ años para el grupo de los casos. El porcentaje de mujeres fue de $46,8 \%$. El universo incluyó a personal vinculado a la Universidad Nacional de Colombia y mayoritariamente a estudiantes universitarios.

Tomando como referencia las unidades de bebida estándar y el volumen de un trago por bebida, en un día habitual de consumo de alcohol los hombres del grupo de casos consumían en promedio $192 \mathrm{~g}$ y las mujeres 244 $\mathrm{g}$, en tanto que los hombres del grupo de control consumían en promedio 23 $\mathrm{g}$ y las mujeres, $37 \mathrm{~g}$.

\section{Expresión del ARNm del SNCA en células mononucleares de sangre}

Debido a algunas limitaciones técnicas, en este ensayo se analizó una muestra de 25 sujetos del grupo control y 25 del grupo de casos. La tasa de expresión se obtuvo mediante los valores de $\mathrm{Cq}$ y se evidenció un incremento en la expresión del ARNm del SNCA en el grupo de casos estadísticamente significativo comparado con el grupo de control $(p=0,0099)$ (figura 1).

La expresión génica por sexos presentó una media de 0,465 en las mujeres del grupo de control y de 1.208 en las del grupo de los casos; en los hombres del grupo de control fue de 0,378 y de 1.346 en los del grupo de los casos.

\section{Cuantificación de la proteína SNCA en plasma}

Se empleó el plasma de 34 sujetos del grupo control y de 27 del grupo de casos. El valor promedio en los niveles de la proteína en plasma fue mayor en el grupo de casos $(199,3 \mathrm{pg} / \mathrm{ml}$ ) que en el de controles $(180,9 \mathrm{pg} / \mathrm{ml})$, sin diferencias estadísticamente significativas entre los dos grupos ( $p=0,7112)$ (figura 2$)$.

\section{Frecuencia de SNV en la región promotora del gen SNCA}

Se evaluaron 31 sujetos del grupo de control y 25 del grupo de casos. En general, no se observaron diferencias estadísticamente significativas entre los grupos en ninguna de las nueve variantes estudiadas en el segmento de la región promotora del SNCA.

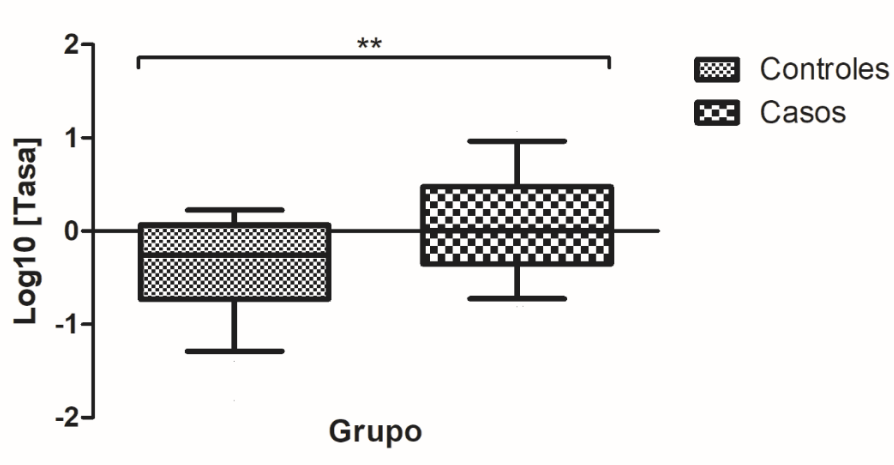

Figura 1. Grado de expresión de ARNm del gen SNCA en células mononucleares de sangre periférica. Los valores se presentan en el diagrama de caja con un rango de percentil de 10 a 90; la línea representa la mediana.

${ }^{* *} p=0,0099$ en la prueba de Mann-Whitney; $n=25$ sujetos en cada grupo

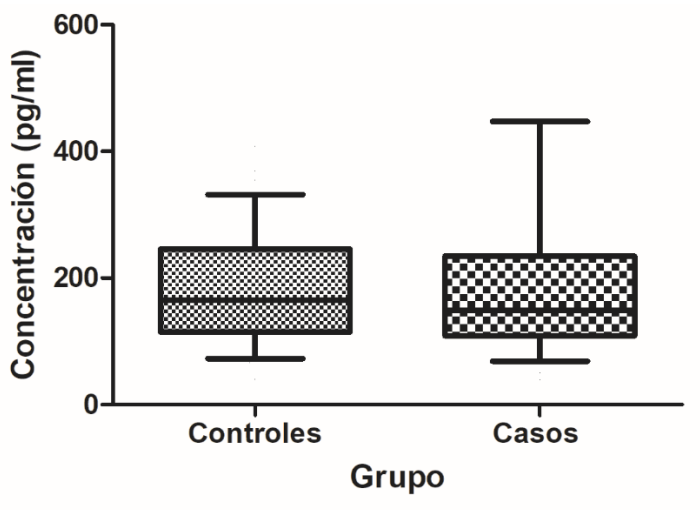

Figura 2. Concentración de la proteína SNCA en plasma sanguíneo. Los valores se presentan en el diagrama de caja con un rango del percentil de 10 a 90; la línea representa la mediana. $p=0,7112$ en la prueba de Mann-Whitney; $n=34$ sujetos en el grupo de control y $n=27$ en el grupo de casos 
La secuenciación de ADN se determinó en la hebra no codificante y, por lo tanto, complementaria de los reportados en las bases de datos. De las variantes previamente reportadas y relacionadas con el consumo de alcohol, se encontró una mayor frecuencia en el genotipo [C/C] para la variante rs2619363 $(\mathrm{G}>\mathrm{C}, \mathrm{T})$, seguido del genotipo heterocigoto $[\mathrm{C} / \mathrm{A}]$, sin registrarse diferencias estadísticamente significativas $(p=0,77)$. Para la variante rs2301134 $(A>G)$ las frecuencias se distribuyeron en los tres genotipos $[C / C],[C / T]$ y $[T / T]$ con mayor frecuencia el genotipo heterocigoto $[\mathrm{C} / \mathrm{T}]$ en los controles, aunque sin diferencias estadísticamente significativas ( $p=0,11)$ (cuadro 1).

Asimismo, las seis variantes previamente identificadas en el segmento analizado, rs542037441 (G>T), rs989496677(C>T), rs927159023 (G>C), rs924048579 $(\mathrm{G}>\mathrm{C})$, rs950036657 (G>A), y rs916862395 $(\mathrm{G}>\mathrm{C}, \mathrm{T})$, resultaron ser monomorfas del tipo homocigoto, [G/G], [C/C], [C/C], [C/C], [G/G] y $[C / C]$, respectivamente, en los dos grupos evaluados y en el $100 \%$ de los participantes en el estudio.

Por otro lado, en el estudio se detectó con gran frecuencia en los dos grupos una SNV en la posición -2171 de la región promotora del gen SNCA, la cual no se encuentra reportada en GenBank (NCBI). Dicha variante se observó con mayor frecuencia en el genotipo heterocigoto [T/A] en los dos grupos, sin diferencia estadísticamente significativa entre ellos $(p=0,14)$.

Cuadro 1. Frecuencia de genotipos y alelos de diferentes SNV de la región promotora del gen SNCA (-1738 y -2299) secuenciados en la hebra no codificante

\begin{tabular}{|c|c|c|c|c|c|c|}
\hline$\overline{\text { SNV }}$ & $\mathbf{n}$ & \multicolumn{3}{|c|}{ Genotipo \% (n) } & \multicolumn{2}{|c|}{ Alelos \% (n) } \\
\hline rs2619363 (-2229) & & $\mathrm{C} / \mathrm{C}$ & $\mathrm{C} / \mathrm{A}$ & $A / A$ & C & A \\
\hline Controles & 27 & $63(17)$ & $33,3(9)$ & $3,7(1)$ & $80(44)$ & $20(11)$ \\
\hline Casos & 24 & $62,5(15)$ & $29,2(7)$ & $8,3(2)$ & $77,1(37)$ & 22,9 (11) \\
\hline$p<0,05$ & & \multicolumn{3}{|c|}{0,77} & \multicolumn{2}{|c|}{0,72} \\
\hline rs542037441 (-2195) & & $\mathrm{C} / \mathrm{C}$ & $\mathrm{C} / \mathrm{A}$ & $\mathrm{A} / \mathrm{A}$ & C & $A$ \\
\hline Controles & 31 & $100(31)$ & 0 & 0 & $100(62)$ & 0 \\
\hline Casos & 25 & $100(25)$ & 0 & 0 & $100(50)$ & 0 \\
\hline $\mathrm{p}<0,05$ & & \multicolumn{3}{|c|}{$>0,99$} & \multicolumn{2}{|c|}{$>0,99$} \\
\hline rs989496677 (-2185) & & $\mathrm{G} / \mathrm{G}$ & $\mathrm{G} / \mathrm{A}$ & $\mathrm{A} / \mathrm{A}$ & $G$ & $A$ \\
\hline Controles & 31 & $100(31)$ & 0 & 0 & $100(62)$ & 0 \\
\hline Casos & 25 & $100(25)$ & 0 & 0 & $100(50)$ & 0 \\
\hline$p<0,05$ & & \multicolumn{3}{|c|}{$>0,99$} & \multicolumn{2}{|c|}{$>0,99$} \\
\hline SNV (-2171) & & $\mathrm{T} / \mathrm{T}$ & $\mathrm{T} / \mathrm{A}$ & $\mathrm{A} / \mathrm{A}$ & $\mathrm{T}$ & A \\
\hline Controles & 31 & $16,1(5)$ & $83,9(26)$ & 0 & $58,1(36)$ & $41,9(26)$ \\
\hline $\begin{array}{l}\text { Casos } \\
p<0,05\end{array}$ & 25 & $4,4(1)$ & $\begin{array}{c}96,0(24) \\
0,14\end{array}$ & 0 & $\begin{array}{r}52(26) \\
0,\end{array}$ & $58(24)$ \\
\hline rs927159023 (-2159) & & $\mathrm{C} / \mathrm{C}$ & $\mathrm{C} / \mathrm{G}$ & $\mathrm{G} / \mathrm{G}$ & C & $G$ \\
\hline Controles & 31 & $100(31)$ & 0 & 0 & $100(62)$ & 0 \\
\hline Casos & 25 & $100(25)$ & 0 & 0 & $100(50)$ & 0 \\
\hline $\mathrm{p}<0,05$ & & & $>0,99$ & & \multicolumn{2}{|c|}{$>0,99$} \\
\hline rs924048519 (-2141) & & $\mathrm{C} / \mathrm{C}$ & $\mathrm{C} / \mathrm{G}$ & $\mathrm{G} / \mathrm{G}$ & C & $G$ \\
\hline Controles & 31 & $100(31)$ & 0 & 0 & $100(62)$ & 0 \\
\hline Casos & 25 & $100(25)$ & 0 & 0 & $100(50)$ & 0 \\
\hline$p<0,05$ & & & $>0,99$ & & \multicolumn{2}{|c|}{$>0,99$} \\
\hline rs2301134 (-2127) & & $\mathrm{C} / \mathrm{C}$ & $\mathrm{C} / \mathrm{T}$ & $\mathrm{T} / \mathrm{T}$ & C & $\mathrm{T}$ \\
\hline Controles & 31 & $19,4(6)$ & $67,7(21)$ & $12,9(4)$ & $53,2(33)$ & $46,8(29)$ \\
\hline Casos & 25 & $32(8)$ & $40(10)$ & $28(7)$ & $52(26)$ & $28(24)$ \\
\hline $\mathrm{p}<0,05$ & & \multicolumn{3}{|c|}{0,11} & \multicolumn{2}{|c|}{0,90} \\
\hline rs950036657 (-2120) & & $\mathrm{C} / \mathrm{C}$ & $\mathrm{C} / \mathrm{T}$ & $\mathrm{T} / \mathrm{T}$ & C & $\mathrm{T}$ \\
\hline Controles & 31 & $100(31)$ & 0 & 0 & $100(62)$ & 0 \\
\hline Casos & 25 & $100(25)$ & 0 & 0 & $100(50)$ & 0 \\
\hline$p<0,05$ & & \multicolumn{3}{|c|}{$>0,99$} & \multicolumn{2}{|c|}{$>0,99$} \\
\hline rs916862395 (-2111) & & $\mathrm{C} / \mathrm{C}$ & $\mathrm{C} / \mathrm{G}$ & $\mathrm{G} / \mathrm{G}$ & C & $\mathrm{G}$ \\
\hline Controles & 31 & $100(31)$ & 0 & 0 & $100(62)$ & 0 \\
\hline Casos & 25 & $100(25)$ & 0 & 0 & $100(50)$ & 0 \\
\hline$p<0,05$ & & \multicolumn{3}{|c|}{$>0,99$} & \multicolumn{2}{|c|}{$>0,99$} \\
\hline
\end{tabular}




\section{Presencia de estreñimiento crónico en la población seleccionada}

Con base en el algoritmo para el diagnóstico del estreñimiento crónico, se encontraron diferencias estadísticamente significativas en cuanto a la presencia de estreñimiento crónico por grupos de consumo de alcohol $(p=0,011)$, observándose un riesgo 4,8 veces mayor de presentar estreñimiento en las personas con problemas de consumo de alcohol (OR=4,8; IC: 1,43-16,2) (figura 3). Se aplicaron los criterios diagnósticos de Roma IV para el síndrome de intestino irritable con el fin de descartar alguna relación entre la presencia de síntomas típicos del estreñimiento crónico y los del síndrome.

\section{Asociación entre el nivel de proteína SNCA y la prevalencia de estreñimiento crónico}

Se evaluaron 34 sujetos en el grupo de control y 27 en el grupo de casos. En general, no se observó una relación entre el diagnóstico de estreñimiento crónico y el consumo de alcohol como factores separados, ni en su interacción sobre el nivel de proteína SNCA $(p=0,982)$.

\section{Asociación entre la expresión del gen SNCA en forma de ARNm y la prevalencia de estreñimiento crónico}

Se analizaron 25 sujetos en cada grupo de estudio. Se observó un incremento de la expresión del SNCA en los sujetos con elevado consumo de alcohol y diagnóstico de estreñimiento crónico (figura 4); no obstante, no se encontró una diferencia estadística significativa para esta interacción (cuadro 2).

\section{Relación entre la expresión del gen SNCA y los SNV}

Se analizaron tres variantes del total de nueve, ya que seis fueron monomorfas en la población estudiada. Según la prueba post hoc de Bonferroni, la SNV en la posición -2171 registró una diferencia estadísticamente significativa en la expresión del SNCA entre los grupos de casos y controles en los sujetos con el genotipo AT ( $p<0,01)$ (figura 5).
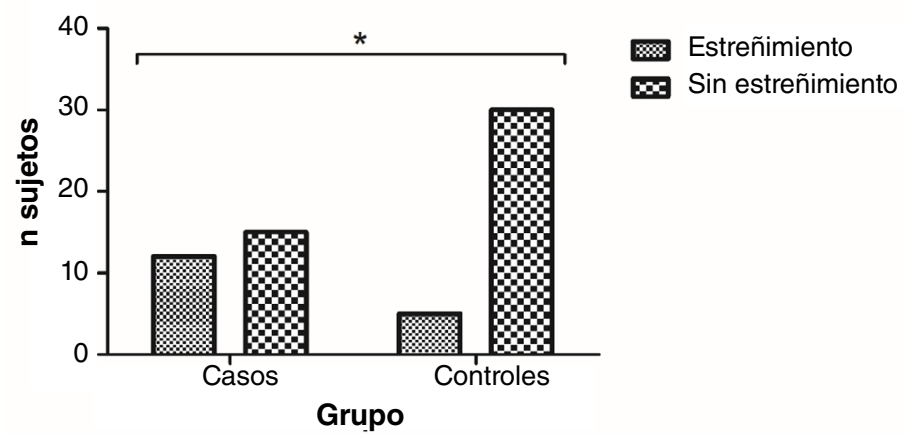

Figura 3. Presencia de estreñimiento crónico en los sujetos de investigación. Los valores corresponden a la frecuencia.

* $p=0,011$ en la prueba exacta de Fisher; $O R=4,8$, (IC95\% 1,43-16,2); grupo de controles: $n=5$ con estreñimiento y $n=30$ sin estreñimiento. Grupo de casos: $n=12$ con estreñimiento y $n=15$ sin estreñimiento

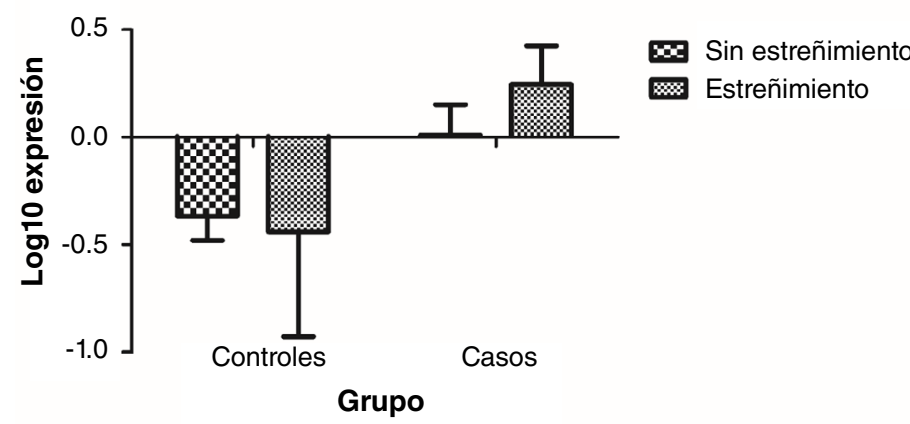

Figura 4. Expresión del gen SNCA según el diagnóstico de estreñimiento crónico. Los valores corresponden a la media. $p=0,460$, en la prueba de ANOVA de dos factores. Grupo de controles: $n=3$ con estreñimiento y $\mathrm{n}=22$ sin estreñimiento. Grupo de casos: $\mathrm{n}=11$ con estreñimiento y $\mathrm{n}=14$ sin estreñimiento

Cuadro 2. Expresión $\left(\log _{10}\right)$ del gen SNCA según el diagnóstico de estreñimiento crónico

\begin{tabular}{lllccc}
\hline & Controles & Casos & Diferencia & Intervalo de confianza & $\mathbf{p}$ \\
\hline Con estreñimiento & $-0,4396$ & 0,2463 & 0,6859 & $-0,1663-1,538$ & $>0,05$ \\
Sin estreñimiento & $-0,3667$ & 0,01003 & 0,3767 & $-0,07064-0,8241$ & $>0,05$ \\
\hline
\end{tabular}


a.

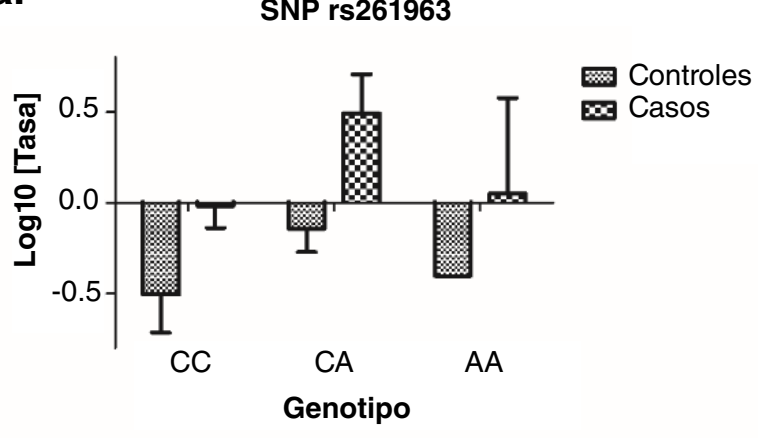

b.

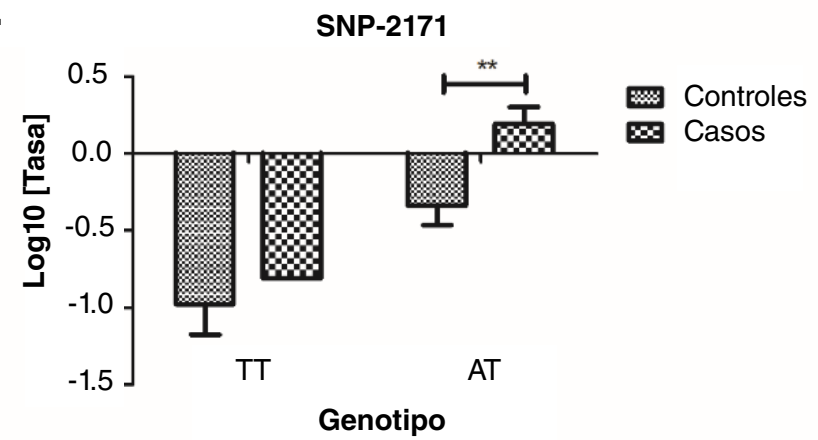

c.

SNP rs2301134

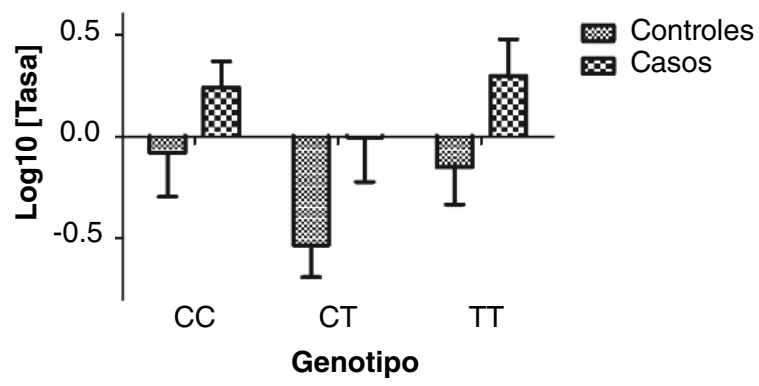

Figura 5. Tasa de expresión del SNCA según el genotipo de tres SNV. Los valores corresponden a la media con su respectiva desviación estándar. a. Tasa de expresión según el genotipo del SNV rs261963, $p=0,920$. b. Tasa de expresión según el genotipo del SNV -2171, $p=0,581$ en la prueba post hoc $y{ }^{* *} p<0,01$ para el genotipo AT en los dos grupos. c. Tasa de expresión según el genotipo del SNV rs2301134, $p=0,875$. $n=34$ sujetos en el grupo de control y $n=27$ en el grupo de casos

\section{Discusión}

En el estudio se utilizó la herramienta AUDIT validada internacionalmente por la Organización Mundial de la Salud (OMS) (32) y, en Colombia, por Campo, et al. (33), y por Ospina, et al. (34). Según la OMS, los criterios de AUDIT establecen que el consumo perjudicial corresponde a $40 \mathrm{~g}$ diarios en mujeres y más de $60 \mathrm{~g}$ diarios en hombres, en tanto que el consumo de dependencia es de más de $60 \mathrm{~g}$ en cualquiera de los sexos (35). Al comparar estos criterios con lo reportado en un día habitual de consumo por los participantes en el estudio, se observó en el grupo de casos un consumo muy superior al establecido.

La expresión de ARNm del gen SNCA en células mononucleares de sangre periférica fue significativamente mayor en el grupo de casos ( $p=0,0099$ ), lo que coincide con lo reportado por Walker, et al. (19), en su evaluación de la expresión de ARNm del gen SNCA mediante microarreglos en primates alcohólicos que consumieron alcohol libremente durante 18 meses, lo que resultó en un incremento 3,21 veces mayor de los niveles de ARNm en sangre periférica en comparación con los controles $(p<0,0001)(19)$.

Es importante tener en cuenta que, en el presente estudio, los sujetos del grupo de casos se abstuvieron de consumir durante las 48 horas previas a la extracción de sangre, a diferencia de los primates no humanos del estudio mencionado, que dispusieron de alcohol hasta el último momento para medir así el efecto crónico y el agudo. Por ello, es posible que, en este caso en particular y bajo las mismas condiciones, el nivel de expresión en humanos se hubiera incrementado. 
En otros estudios con humanos, se ha evaluado la expresión génica del SNCA en sangre con un periodo de abstinencia de 24 a 72 horas y se ha registrado un aumento significativo de la expresión en el grupo de las personas con alcoholismo ( $p=0,021)(17) y$, aunque el estudio de Bönsch, et al. (36), se hizo únicamente en hombres y en el presente en el grupo de casos había 17 hombres y ocho mujeres, se observó un aumento de la expresión de 1,1 veces en los hombres comparados con las mujeres.

Por otro lado, aunque la concentración de la proteína en plasma aumentó en el grupo de casos $(199,3 \mathrm{pg} / \mathrm{ml})$ con respecto al de control $(180,9 \mathrm{pg} / \mathrm{ml})$, las diferencias no fueron estadísticamente significativas $(p=0,07112)$, lo que contrasta con lo evidenciado por Bönsch, et al., quienes reportaron un aumento significativo de la proteína en personas con abstinencia de 24 a 72 horas $(14,33$ $\mathrm{ng} / \mathrm{ml})$, comparadas con los controles sanos $(5,92 \mathrm{ng} / \mathrm{ml})(\mathrm{p}<0,0001)(36)$.

En el presente estudio, el incremento en la concentración de la proteína no fue estadísticamente significativo comparado con los niveles de expresión del ARNm del SNCA por varias razones: en primer lugar, las determinaciones se realizaron en compartimentos diferentes, el ARNm en lisados de células mononucleares de sangre periférica y la proteína en plasma, por lo que no necesariamente tendrían que ser concordantes, como se ha demostrado en diferentes tejidos y regiones cerebrales $(4,8,9)$. En segundo lugar, las diferencias podrían deberse a mecanismos de regulación postranscripcionales que impiden la traducción eficaz del ARNm $(37,38)$; tan es así que los cambios postranscripcionales del ARNm del SNCA están regulados por la presencia de varios microARN, especialmente el mir-7 y el mir-153, que se unen a la región 3'-UTR del SNCA y cuya expresión es inversamente proporcional a la expresión del ARNm y la proteína $(39,40)$.

En cuanto a las nueve variantes identificadas de nucleótido único, los hallazgos previos han informado de una asociación entre la presencia de la variante rs2619363 y el fenotipo de deseo compulsivo de alcohol (craving) $(p=0,01)$; sin embargo, en el presente estudio no se observó dicha diferencia $(p=0,77)$. En cuanto a la variante rs2301134, aquí no se encontraron diferencias entre las personas en abstinencia durante 48 horas como mínimo y los sujetos de control $(p=0,11)$, al igual que se registró en el estudio de Foroud, et al., quienes no encontraron relación entre este SNV y el fenotipo de deseo compulsivo de alcohol $(p=0,69)$ tan estrechamente vinculado con la dependencia (21), el cual se da en periodos de abstinencia. Las variantes monomorfas identificadas en la presente muestra tuvieron una frecuencia similar a la reportada mundialmente, sin evidencia de una posible vinculación con el consumo de alcohol.

Por otro lado, en el presente estudio se identificó una SNV en la posición -2171 del promotor del gen SNCA con una mayor frecuencia del genotipo heterocigoto $[A / T]$ en los dos grupos evaluados. Esta SNV no se encuentra reportada en GenBank (NCBI) como variante, por lo que su análisis puede ser de utilidad para futuras investigaciones. Esta variante de SNV en -2171 presentó una diferencia significativa para el genotipo heterocigoto $[A / T]$, observándose un incremento de la expresión génica de ARNm en el grupo de los casos $(p<0,01)$. No se encontraron estudios previos que evaluaran la relación entre la expresión del gen y las SNV mencionadas en el promotor. Es importante recordar que el promotor es la región del gen donde se ensambla la maquinaria de la transcripción y que los cambios en los nucleótidos pueden aumentar o disminuir la afinidad por los factores proteicos 
participantes y, por lo tanto, afectar la tasa de transcripción. En este caso, la SNV -2171 [A/T] podría estar relacionada con ese aumento en la expresión de SNCA en el grupo de casos.

Por otro lado, la presencia de estreñimiento se asoció con un riesgo 4,8 veces mayor en sujetos con problemas de consumo de alcohol $(p=0,011)$; sin embargo, no se evidenció relación con la sobreexpresión de SNCA. Debe tenerse en cuenta que los estudios previos se hicieron en un modelo animal con modificación genética para sobreexpresar ARNm e incrementar los niveles proteicos de SNCA (24-30). En dichos modelos animales, se ha observado una reducción en el número de bolas fecales, un aumento de 2,2 veces en el tiempo de expulsión, un contenido fecal 2,9 veces mayor y una alteración de los estímulos para la defecación correspondiente al estreñimiento $(27,28,41,42)$, que posiblemente se relacionen con lo postulado por Sharma, et al., en el sentido de que la presencia del estreñimiento se explicaría por la disbiosis que afecta el epitelio del colon, lo que permite que los agregados de SNCA puedan infiltrarse a través del sistema nervioso entérico hasta llegar al sistema nervioso central (43).

En el presente estudio observacional, se evidenció una diferencia en la expresión del ARNm del SNCA mas no en la proteína, que por su acumulación y agregación causa los efectos motores y no motores (entre ellos el estreñimiento) ya descritos para las llamadas alfa sinucleopatías. Por las tasas de transcripción observadas es poco probable que existan agregados de SNCA (se requieren grandes concentraciones) que puedan infiltrarse en el epitelio intestinal y causar síntomas relacionados con el estreñimiento.

Hasta donde se sabe, este es el primer estudio en el que se intenta evidenciar esta relación, por lo que se requeriría más investigación in vivo e in vitro de tipo observacional y de intervención, con diseños experimentales orientados a determinarla.

El consumo crónico de alcohol tiene manifestaciones psiquiátricas, psicológicas y biológicas similares a otras enfermedades neurodegenerativas. En este contexto, los resultados del presente estudio no concuerdan con lo observado en los pacientes con enfermedad de Parkinson, en la que también se presentan niveles incrementados de la SNCA y el estreñimiento es frecuente, generalmente antes de que se manifiesten los síntomas clínicos propios de la enfermedad, por lo que se ha asociado la frecuencia de evacuaciones intestinales con el riesgo futuro de padecerla; se ha encontrado que aquellas personas con menos de una evacuación por día presentan un riesgo de 2,7 a 4,5 veces mayor (44) y que quienes presentan estreñimiento como antecedente tienen un estadio más grave de la enfermedad (45). Sin embargo, aunque tanto en la enfermedad de Parkinson como en el alcoholismo, hay incremento de SNCA y daños cerebrales, es posible que la presencia de estreñimiento en la enfermedad de Parkinson se deba a otros factores diferentes a los del alcoholismo aún no determinados y que no se relacionen con cambios en la permeabilidad del intestino y en la biota intestinal por acción del alcohol, como se ha empezado a esclarecer (46), y se relacionan más con otras alteraciones en las vías dopaminérgicas que tienen un papel importante en la motilidad intestinal.

En este estudio, se evidenció la sobreexpresión de ARNm del gen SNCA en las células mononucleares de los sujetos con problemas de consumo de alcohol, pero no se encontró una relación con la concentración plasmática de la proteína. Además, en el grupo con alto consumo de alcohol, se registró un 
mayor riesgo de presentar estreñimiento, aunque sin relación con la expresión diferencial de ARNm del SNCA. Por otro lado, no se observó una diferencia estadísticamente significativa en la frecuencia de las SNV y se identificó una en la posición -2171, no reportada previamente como variante en GenBank, y cuyo genotipo $[A / T]$ parece contribuir al incremento de la expresión génica de SNCA.

\section{Agradecimientos}

A los participantes voluntarios del estudio, a los estudiantes de pregrado y posgrado del Semillero de Investigación, subgrupo de Genética de las Adicciones, Grupo de Genética Clínica, y a todo el personal del Instituto de Genética de la Universidad Nacional de Colombia que de una u otra forma han contribuido al desarrollo de este trabajo.

\section{Referencias}

1. Organización Mundial de la Salud. Estrategia mundial para reducir el uso nocivo del alcohol. Ginebra: Organización Mundial de la Salud; 2010. p. 1-46. Fecha de consulta: 5 de enero de 2018. Disponible en: https://www.who.int/substance abuse/activities/msbalcstrategyes.pdf

2. Observatorio de Drogas de Colombia. Estudio de consumo de sustancias psicoactivas en Colombia, 2013. p. 1-182. Fecha de consulta: 5 de enero de 2018. Disponible en: https://www. unodc.org/documents/colombia/2014/Julio/Estudio de Consumo UNODC.pdf

3. Serecigni J. Neurobiología del alcoholismo. Psicología desde el Caribe. 2013;30:21-35.

4. Janeczek P, Lewohl J. The role of a-synuclein in the pathophysiology of alcoholism. Neurochem Int. 2013;63:154-62. https://doi.org/10.1016/j.neuint.2013.06.007

5. Swant J, Goodwin J, North A, Ali A, Gamble J, Chirwa S, et al. a-synuclein stimulates a dopamine transporter-dependent chloride current and modulates the activity of the transporter. J Biol Chem. 2011;286:43933-43. https://doi.org/10.1074/jbc.M111.241232

6. Liang T, Carr LG. Regulation of alpha-synuclein expression in alcohol-preferring and -non preferring rats. J Neurochem. 2006;99:470-82. https://doi.org/10.1111/j.14714159.2006.04111.x

7. Butler B, Saha K, Rana T, Becker JP, Sambo D, Davari P, et al. Dopamine transporter activity is modulated by a-synuclein. J Biol Chem. 2015;290:29542-54. https://doi.org/10.1074/jbc.M115.691592

8. Sui Y, Bullock K, Erickson M, Zhang J, Banks W. Alpha synuclein is transported into and out of the brain by the blood-brain barrier. Peptides. 2014;62:197-202. https://doi.org/10.1016/j.peptides.2014.09.018

9. Lööv C, Scherzer CR, Hyman BT, Breakefield XO, Ingelsson M. a-synuclein in extracellular vesicles: Functional implications and diagnostic opportunities. Cell Mol Neurobiol. 2016;36:437-48. https://doi.org/10.1007/s10571-015-0317-0

10. Simonsen A, Kuiperij B, El-Agnaf A, Omar M, Engelborghs S, Herukka S-K, et al. The utility of $\alpha$-synuclein as biofluid marker in neurodegenerative diseases: A systematic review of the literature. Biomark Med. 2016;10:19-34. https://doi.org/10.2217/BMM.14.105

11. Omar M, Agnaf E, Salem S, Paleologou K, Cooper L, Fullwood N, et al. Synuclein implicated in Parkinson's disease is present in extracellular biological fluids including human plasma. FASEB J. 2003;17:1945-7. https://doi.org/10.1096/fj.03-0098fje

12. Henchcliffe C. Blood and cerebrospinal fluid markers in Parkinson's disease: Current biomarker findings. Curr Biomark Find. 2015;5:1-11. https://doi.org/10.2147/CBF.S50424

13. Nakai M, Fujita M, Waragai M, Sugama S, Wei J, Akatsu H, et al. Expression of a-synuclein, a presynaptic protein implicated in Parkinson's disease, in erythropoietic lineage. Biochem Biophys Res Commun. 2007;358:104-10. https://doi.org/10.1016/j.bbrc.2007.04.108

14. Abd-Elhadi S, Honig A, Simhi-Haham D, Schechter M, Linetsky E, Ben-Hur T, et al. Total and proteinase K-resistant $\alpha$-synuclein levels in erythrocytes, determined by their ability to bind phospholipids, associate with Parkinson's disease. Sci Rep. 2015;5:1-12. https://doi.org/10.1038/srep11120

15. Kang W, Chen W, Yang Q, Zhang L, Zhang L, Wang X, et al. Salivary total a-synuclein, oligomeric $\alpha$-synuclein and SNCA variants in Parkinson's disease patients. Sci Rep. 2016;6:1-8. https://doi.org/10.1038/srep28143 
16. Wang X, Yu S, Li F, Feng T. Detection of $\alpha$-synuclein oligomers in red blood cells as a potential biomarker of Parkinson's disease. Neurosci Lett. 2015;599:115-9. https://doi.org/10.1016/j.neulet.2015.05.030

17. Binsch D, Reulbach U, Bayerlein K, Hillemacher T, Kornhuber J, Bleich S. Elevated alpha synuclein mRNA levels are associated with craving in patients with alcoholism. Biol Psychiatry. 2004;56:984-6. https://doi.org/10.1016/j.biopsych.2004.09.016

18. Ziolkowska B, Gieryk A, Wawrzczak-Bargiela A, Krowka T, Kaminska D, Korkosz A, et al. a-Synuclein expression in the brain and blood during abstinence from chronic alcohol drinking in mice. Neuropharmacology. 2008;54:1239-46. https://doi.org/10.1016/j.neuropharm.2008.04.001

19. Walker S, Grant K. Peripheral blood $\alpha$-synuclein mRNA levels are elevated in cynomolgus monkeys that chronically self-administer ethanol. Alcohol. 2006;38:1-4. https://doi.org/10.1016/j.alcohol.2006.03.008

20. Bönsch D, Lederer T, Reulbach U, Hothorn T, Kornhuber J, Bleich S. Joint analysis of the NACP-REP1 marker within the alpha synuclein gene concludes association with alcohol dependence. Hum Mol Genet. 2005;14:967-71. https://doi.org/10.1093/hmg/ddi090

21. Foroud T, Wetherill LF, Liang T, Dick DM, Hesselbrock V, Kramer J, et al. Association of alcohol craving with a-synuclein (SNCA). Alcohol Clin Exp Res. 2007;31:537-45. https://doi.org/10.1111/j.1530-0277.2007.00337.x

22. Braak H, Rüb U, Gai WP, Del Tredici K. Idiopathic Parkinson's disease: Possible routes by which vulnerable neuronal types may be subject to neuroinvasion by an unknown pathogen. J Neural Transm. 2003;110:517-36. https://doi.org/10.1007/s00702-002-0808-2

23. Shannon KM, Keshavarzian A, Dodiya HB, Jakate S, Kordower JH. Is alpha-synuclein in the colon a biomarker for premotor Parkinson's disease? Evidence from 3 cases. Mov Disord. 2012;27:716-9. https://doi.org/10.1002/mds.25020

24. Chesselet MF, Richter F, Zhu C, Magen I, Watson MB, Subramaniam SR. A progressive mouse model of Parkinson's disease: The Thy1-aSyn ("Line 61") mice. Neurotherapeutics. 2012;9:297-314. https://doi.org/10.1007/s13311-012-0104-2

25. Rockenstein E, Mallory M, Hashimoto M, Song D, Shults CW, Lang I, et al. Differential neuropathological alterations in transgenic mice expressing a-synuclein from the plateletderived growth factor and Thy-1 promoters. J Neurosci Res. 2002;68:568-78. https://doi.org/10.1002/jnr.10231

26. Verbaan D, Marinus J, Visser M, Van Rooden SM, Stiggelbout AM, van Hilten JJ. Patientreported autonomic symptoms in Parkinson disease. Neurology. 2007;69:333-41. https://doi.org/10.1212/01.wnl.0000266593.50534.e8

27. Wang L, Magen I, Yuan PQ, Subramaniam SR, Richter F, Chesselet MF, et al. Mice overexpressing wild-type human alpha-synuclein display alterations in colonic myenteric ganglia and defecation. Neurogastroenterol Motil. 2012;24:1-12. https://doi.org/10.1111/j.1365-2982.2012.01974.x

28. Sampson TR, Debelius JW, Thron T, Janssen S, Shastri GG, Ilhan ZE, et al. Gut microbiota regulate motor deficits and neuroinflammation in a model of Parkinson's disease. Cell. 2016;167:1469-80.e12. https://doi.org/10.1016/j.cell.2016.11.018

29. Engen P, Green S, Voigt R, Forsyth C, Keshavarzian A. The gastrointestinal microbiome: Alcohol effects on the composition of intestinal microbiota. Alcohol Res. 2015;37:223-36. https://doi.org/10.13140/RG.2.1.4342.9285

30. Yan A, Fouts D, Brandl J, Starkel P, Torralba M, Schott E, et al. Enteric dysbiosis associated with a mouse model of alcoholic liver disease. Hepatology. 2011;53:96-105. https://doi.org/10.1002/hep.24018

31. Domingo J. The new Rome criteria (IV) of functional digestive disorders in clinical practice. Med Clin (Barc). 2017;148:464-8. https://doi.org/10.1016/j.medcli.2016.12.020

32. Babor T, Higgins J, Saunders J, Monteiro M. Cuestionario de identificación de los transtornos debidos al consumo de alcohol. Ginebra: Organización Mundial de la Salud; 2001. p. 1-40.

33. Campo A, Villamil M, Herazo E. Confiabilidad y dimensionalidad del AUDIT en estudiantes de medicina. Psicología desde el Caribe. 2013;30:21-35.

34. Ospina J, Manrique F, Ariza N. Confiabilidad y dimensionalidad del cuestionario para identificación de trastornos debidos al consumo de alcohol (AUDIT) en estudiantes universitarios de Tunja (Colombia). Salud Uninorte. 2012;28:276-82. 
35. Anderson P, Gual L, Colón J. Alcohol y atención primaria de la salud alcohol y atención primaria de la salud. Washington, D.C.: Organización Panamericana de la Salud; 2008.

36. Bönsch D, Greifenberg V, Bayerlein K, Biermann T, Reulbach U, Hillemacher T, et al. $\alpha$-synuclein protein levels are increased in alcoholic patients and are linked to craving. Alcohol Clin Exp Res. 2005;29:763-5. https://doi.org/10.1097/01.ALC.0000164360.43907.24

37. Sheth U, Parker R. Decapping and decay of messenger RNA occur in cytoplasmic processing bodies. Science. 2003;300:805-8. https://doi.org/10.1126/science.1082320

38. Parker R, Sheth U. P bodies and the control of mRNA translation and degradation. Mol Cell. 2007;25:635-46. https://doi.org/10.1016/j.molcel.2007.02.011

39. Doxakis E. Post-transcriptional regulation of a-synuclein expression by mir-7 and mir-153. J Biol Chem. 2010;285:12726-34. https://doi.org/10.1074/jbc.M109.086827

40. McMillan K, Murray T, Bengoa N, Cordero O, Cooper J, Buckley A, et al. Loss of microRNA-7 regulation leads to $\alpha$-synuclein accumulation and dopaminergic neuronal loss in vivo. Mol Ther. 2017;25:2404-14. https://doi.org/10.1016/j.ymthe.2017.08.017

41. Wang L, Fleming S, Chesselet M, Taché Y. Abnormal colonic motility in mice overexpressing human wild-type a-synuclein. Neuroreport. 2008;19:873-6. https://doi.org/10.1097/WNR.0b013e3282ffda5e

42. Kuo Y, Li Z, Jiao Y, Gaborit N, Pani A, Morrison B, et al. Extensive enteric nervous system abnormalities in mice transgenic for artificial chromosomes containing Parkinson diseaseassociated alpha-synuclein gene mutations precede central nervous system changes. Hum Mol Genet. 2010;19:1633-50. https://doi.org/10.1097/WNR.0b013e3282ffda5e

43. Sharma A, Kurek J, Morgan JC, Wakade C, Rao SS. Constipation in Parkinson's disease: A nuisance or nuanced answer to the pathophysiological puzzle? Curr Gastroenterol Rep. 2018;20:1. https://doi.org/10.1007/s11894-018-0609-x

44. Abbott R, Petrovitch H, White L, Masaki K, Tanner C, Curb J, et al. Frequency of bowel movements and the future risk of Parkinson's disease. Neurology. 2001;57:456-62. https://doi.org/10.1212/WNL.58.5.838-a

45. Rodríguez O, Torres L, Meza K, López R, Ruiz H, Cosentino C. Estreñimiento como factor asociado a mayor severidad en pacientes con enfermedad de Parkinson del Instituto Nacional de Ciencias Neurológicas del Perú. Diagnóstico. 2019;57:180-3. https://doi.org/10.33734/diagnostico.v57i4.169

46. Leclercq S, De Timary P, Delzenne N, Stärkel P. The link between inflammation, bugs, the intestine and the brain in alcohol dependence. Transl Psychiatry. 2017;7:e1048. https://doi.org/10.1038/tp.2017.15 